Enfermagem Brasil 2018;17(2):107-13

\title{
ARTIGO ORIGINAL \\ Protocolo gerenciado de sepse e seus respectivos resultados em um hospital privado na cidade do Rio do Janeiro
}

Adriana do Prado Rodrigues Carneiro*, Flavia Rabelo Santos**, Sallon Carneiro dos Santos ${ }^{\star \star *}$, André Nobre de Oliveira, M.Sc. ${ }^{* * * *}$, Beatriz Gerbassi Costa Aguiar, D.Sc. ${ }^{* * * *}$

*Pós-graduanda em Enfermagem Hospitalar com ênfase em saúde do idoso no formato de residência, pela FADBA, Enfermeira Plantonista da Unidade de Internação do Hospital Adventista Silvestre, Unidade Itaboraí, Rio de Janeiro, ${ }^{* *}$ Pós-graduanda em Enfermagem Hospitalar com ênfase em saúde do idoso no formato de residência, pela FADBA, Enfermeira Plantonista da Unidade de Internação do Hospital Adventista Silvestre, Unidade Cosme Velho, Rio de Janeiro, ${ }^{* * *}$ Pós-graduando em Urgência e Emergência e UTI pela FADBA, Enfermeiro Plantonista da Unidade de Terapia Intensiva do Hospital Adventista Silvestre, Unidade Itaboraí, Rio de Janeiro, ${ }^{* * * *}$ Pós-graduando em Docência do Ensino Superior, pelo UNASP, Mestrando em Enfermagem pela Universidade Federal do Estado do Rio de Janeiro (UNIRIO), Gerente de Enfermagem do Hospital Adventista Silvestre, Unidade Cosme Velho, Rio de Janeiro, *****Professora Associada do Dpto de Enfermagem Médico-Cirúrgica da Escola de Enfermagem Alfredo Pinto da Universidade Federal do Estado do Rio de Janeiro e Professora do Programa de Mestrado em Enfermagem da Universidade Federal do Estado do Rio de Janeiro, Rio de Janeiro

Recebido em 23 de setembro de 2017; aceito em 13 de dezembro de 2017.

Endereço de correspondência: André Nobre de Oliveira, Depto de Enfermagem do Hospital Adventista Silvestre, Ladeira do Ascurra, 274, Cosme Velho, 22241-220 Rio de Janeiro RJ, Email: andre.nobre@hasilvestre.org.br; Adriana do Prado Rodrigues Carneiro: adriana.pradoefmg@hotmail.com; Flavia Ravelo dos Santos: flavia-lanna@hotmail.com; Sallon Carneiro dos Santos: sallon.carneiro@gmail.com; Beatriz Gerbassi Costa Aguiar: residenfermagem@unirio.br

\section{Resumo}

Objetivo: Consistiu em avaliar o protocolo gerenciado de sepse e seus respectivos resultados em um hospital privado na cidade do Rio de Janeiro. Métodos: Estudo do tipo documental, dados coletados por meio da análise de 295 protocolos em um hospital privado na cidade do Rio de Janeiro. Resultados: Dos protocolos de sepse abertos no período de um ano, $15 \%$ foram abertos como SIRS, $41 \%$ como sepse e $44 \%$ como sepse grave, $50 \%$ destes foram abertos na Emergência com diagnósticos de sepse de origem comunitária. Ao avaliar o índice de mortalidade por sepse, obteve-se um resultado de $35 \%$ de óbitos, mantendo sua taxa abaixo da taxa nacional que é de $65 \%$, ficando dentro da média mundial de $30 \%-40 \%$. Evoluíram para alta hospitalar $65 \%$ dos casos. Conclusão: O protocolo de sepse tem um alto nível de evidência e sua existência aumenta o sucesso terapêutico no que se refere ao baixo índice de óbitos por sepse comparados ao índice nacional.

Palavras-chave: sepse, infecção, controle, mortalidade.

\section{Abstract \\ Sepsis management protocol and outcomes in a private hospital in the city of Rio de Janeiro}

Aim: The objective was to evaluate the sepsis management protocol and outcomes in a private hospital in the city of Rio de Janeiro. Methods: This is a documentary study, which collected data using 295 protocols. Results: We found the following results from the sepsis protocols during a year: $15 \%$ were diagnosed as SIRS, $41 \%$ as sepsis and $44 \%$ as severe sepsis, $50 \%$ of these were from the emergency room with diagnoses of sepsis of community acquired. When evaluating the sepsis mortality rate, a result of $35 \%$ of deaths was obtained, keeping its rate below the national rate of $65 \%$, being within the world average of $30 \%-40 \%$, and $65 \%$ were discharge from hospital. Conclusion: The Sepsis protocol has a high level of evidence and it improves the therapeutic success with regard to the low rate of sepsis deaths compared to the national rate. 
Key-words: sepsis, infection, control and mortality.

\section{Resumen \\ El protocolo de Sepse y sus respectivos resultados en un hospital privado en la ciudad de Río de Janeiro}

Objetivo: Evaluar el protocolo de sepsis y sus respectivos resultados en un hospital privado en la ciudad de Río de Janeiro. Método: Estudio tipo documental. Los datos fueron recolectados a través del análisis de 295 protocolos. Resultados: Los resultados encontrados de los protocolos de sepsis en el período de un año fueron: el $15 \%$ fue diagnosticado como SIRS, el $41 \%$ como sepsis y el $44 \%$ como sepsis grave, $50 \%$ de éstos fueron diagnosticados en la Emergencia como sepsis adquirida en la comunidad. Al evaluar el índice de mortalidad por sepsis, se obtuvo un resultado de $35 \%$ de muertes, manteniendo su tasa por debajo de la media nacional que es del $65 \%$ y dentro del promedio mundial del $30 \%-40 \%$. El $65 \%$ de los casos evaluados recibieron el alta hospitalaria. Conclusión: El protocolo de Sepse tiene un alto nivel de evidencia y su existencia aumenta el éxito terapéutico en lo que se refiere al bajo índice de óbitos por sepsis comparados al índice nacional.

Palabras-clave: sepse, infección, control y mortalidad.

Introdução

A sepse se manifesta com um largo espectro de gravidades, dependendo do tempo decorrido desde suas primeiras manifestações. Quando a sepse progride para disfunção de um ou mais órgãos é chamada de sepse grave, e choque séptico se há hipotensão que não responde a reposição fluida agressiva [1].

A sepse representa um importante problema de saúde pública no Brasil, com elevados índices de mortalidade, alta prevalência, tanto nos serviços públicos quanto privados e altos custos de tratamento. Considerando o ponto de vista econômico, a sepse representa enormes dispêndios para o sistema de saúde, assim como uma significante perda de produtividade oriunda de longas permanências hospitalares e altas taxas de mortalidade [2].

A incidência de sepse grave aumentou $91,3 \%$ nos últimos 10 anos. Ela ocorre de 1 a 3 casos por 1000 indivíduos na população em diferentes regiões do mundo, e se associa com elevadas taxas de mortalidade, que permaneceram inalteradas no período [2]. No Brasil, $25 \%$ dos pacientes hospitalizados atendem aos critérios diagnósticos de sepse grave ou choque séptico, com taxas progressivamente maiores de mortalidade devida a sepse $(34,7 \%)$, sepse grave $(47,3 \%)$ e choque séptico $(52,2 \%)[1]$.

Os graus de gravidade da síndrome séptica na sepse, sepse grave e choque séptico representam a evolução temporal de uma mesma doença. A identificação precoce da sepse é, portanto, o passo mais importante para aumentar os efeitos positivos do melhor tratamento. Portanto, é necessário adotar estratégias hospitalares abrangentes de triagem que permitam a identificação dos pacientes hospitalizados com sepse na fase inicial da doença. $O$ retardamento do tratamento da sepse pode comprometer gravemente o prognóstico [3,4].

Medidas preventivas, assim como a implantação de protocolos para detecção e tratamento precoce, são passos voltados para a diminuição das taxas de morbidade e mortalidade, e dos custos associados à sepse [5]. Os protocolos são documentos científicos que vinculam e orientam a assistência médico-farmacêutica, sistematizando o padrão do manejo clínico para determinado problema de saúde [5].

As diretrizes internacionais de sepse de 2016 mostram que ao avaliar um paciente com suspeita de infecção fora da Unidade de Terapia Intensiva, o médico deve procurar pelas variáveis do qSOFA Score (GCS < 15, FR $\geq 22$ e PAS $\leq 100$ ). Caso o paciente preencha 2 ou mais critérios do qSOFA Score, a árvore diagnóstica continua. Exames laboratoriais devem ser coletados para que o SOFA Score seja calculado. Um SOFA Score que demonstre aumento de 2 ou mais pontos leva à confirmação do diagnóstico de sepse. Se esse paciente tiver a necessidade de uso de vasopressor para manter uma pressão arterial média maior que 65 $\mathrm{mmHg}$ e se o nível de lactato for $>2 \mathrm{mmol} / \mathrm{L}$ mesmo após reanimação volêmica adequada, ele se encaixa no diagnóstico de choque séptico [6,7].

Diante desse contexto, o presente estudo justifica-se por mostrar que a sepse apesar de ser um problema de saúde publica no país, através do gerenciamento dos cuidados frente ao paciente com sepse, esta realidade pode ser mudada. O uso dos protocolos clínicos hospitalares está sendo cada vez maior, eles auxiliam a equipe multiprofissional na tomada de decisões, sejam elas simples ou complexas. O surgimento de equipamentos cada vez mais 
modernos tem deixado os profissionais de saúde a mercê de uma gama variabilidade em suas condutas, trazendo resultados não muito agradáveis à assistência ao paciente.

Desse modo, o presente estudo traz como objetivo avaliar o protocolo gerenciado de sepse e discutir os resultados em um hospital privado do Rio de Janeiro, uma vez que se configuram como diretrizes assistenciais que monitoram continuamente os indicadores de qualidade da prática clínica, visando garantir segurança e qualidade na assistência ao paciente. A maior relevância do mesmo é por contribuir na sistematização do atendimento ao paciente, orientando o trabalho padronizado de profissionais qualificados na assistência e minimizando a variação de cuidados especializados.

\section{Material e métodos}

O estudo realizado foi do tipo documental com abordagem quantitativo. Foram analisados o processo de implantação do protocolo gerenciado de sepse e seus respectivos resultados em um hospital privado na cidade do Rio de Janeiro, no período de julho de 2015 a julho de 2016.

Os dados foram coletados em um hospital privado na cidade do Rio de Janeiro, por meio da análise de 295 protocolos de sepse e tabulados através de tabelas e gráficos no Microsoft Excel. Os critérios de inclusão da pesquisa foram todos os protocolos de sepse abertos no período do ano de 2015 e 2016 que estavam com desfecho clínico fechado como: SIRS, Sepse, Sepse grave e Choque séptico. Os critérios de exclusão foram os protocolos abertos fora do período mencionado e aqueles protocolos sem desfecho clínico definido.

O estudo pode ser definido como estando livre de riscos, uma vez que foi trabalhado apenas com dados secundários, não envolvendo seres humanos de forma direta na pesquisa. Vale salientar que o presente estudo atendeu as recomendações previstas na resolução 466/12 do Ministério da Saúde. Atendendo aos princípios e aspectos éticos o projeto de pesquisa foi submetido e aprovado pelo Comitê de Ética em Pesquisa da Faculdade Adventista da Bahia, com Certificado de Aceite e Apreciação Ética n 1.807.097.

\section{Resultados e discussão}

A sepse permanece como um grande desafio em todo o mundo e não é diferente em nosso país. O estudo agrupou um número significativo de protocolos de sepse, abertos durante um ano em um hospital de grande porte do Rio de Janeiro, e analisou 295 fichas, distribuídas com seus respectivos desfechos, SIRS, Sepse e Sepse grave. A Síndrome da Resposta Inflamatória Sistêmica (SIRS) é a reação inflamatória desencadeada pelo organismo frente a qualquer agressão infecciosa ou não infecciosa. A sepse é uma disfunção de órgãos com risco de vida causada por uma resposta do hospedeiro à infecção desregulada. Sepse grave é caracterizada pela presença dos critérios de sepse associada à disfunção orgânica ou sinais de hipoperfusão [9]. Em um estudo publicado pela Revista JAMA (The Third Internacional Consensus Definitions for Sepsis and Septic Shock-Sepsis) as definições foram atualizadas para sepse e choque séptico.

Percebeu-se que há um número significativo de protocolos abertos apenas com sinais de SIRS, totalizando $15 \%$, o que nos serve como um indicador de qualidade ter em uma instituição hospitalar o gerenciamento de um protocolo tão significante na prática clínica dos profissionais, o que proporciona a identificação precoce dos sinais de qualquer alteração sistêmica de cada indivíduo.

Ao longo de mais de uma década, depois da divulgação dos benefícios da terapia precoce guiada por metas e da publicação da primeira diretriz da Campanha de Sobrevivência à Sepse (CSS) em 2004, surgiram várias evidências sobre a importância do tratamento precoce na diminuição da mortalidade em pacientes com sepse grave ou choque séptico [1011]. Ao longo do tempo, a identificação dos itens com melhor associação com a redução da mortalidade permitiu simplificar as intervenções iniciais no paciente com sepse grave ou choque séptico, enfatizando-se a antibioticoterapia adequada (hemocultura antes do antibiótico + antibiótico de amplo espectro em 1 hora) e o controle da instabilidade hemodinâmica (30 $\mathrm{mL} / \mathrm{kg}$ de cristaloides para pressão arterial média $<65 \mathrm{mmHg}$ ou lactato $\geq 4 \mathrm{mmol} / \mathrm{L}+$ vasopressores para hipotensão refratária a volume) [10]. 


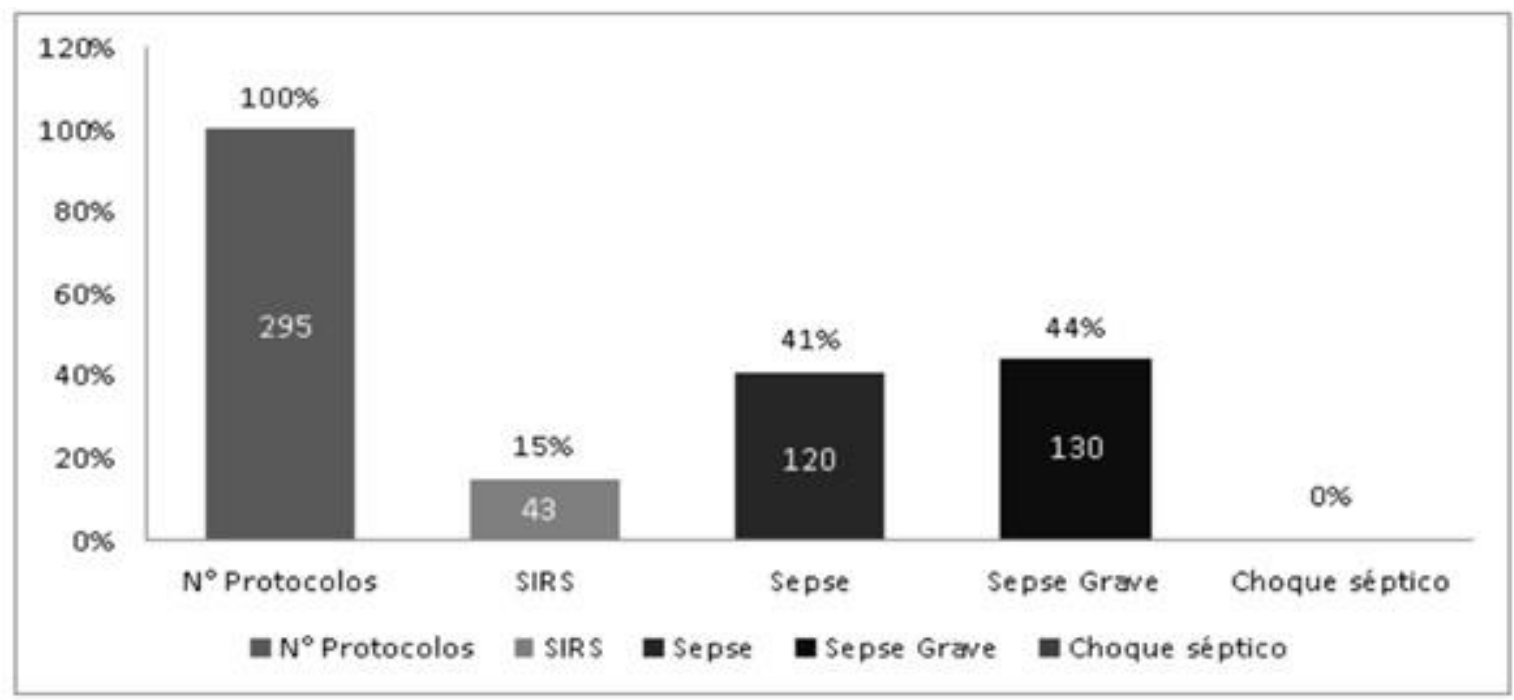

Fonte: Autor.

Figura 1 - Quantitativo de SIRS, Sepse e Sepse grave de um hospital de grande porte na cidade do Rio de Janeiro, julho de 2015 a julho de 2016.

No Brasil, o quadro de sepse é alarmante. Dados de estudos epidemiológicos, coordenados pelo Instituto Latino Americano de Sepse (ILAS) apontam que cerca de $30 \%$ dos leitos das instituições hospitalares em nosso país são ocupados por pacientes com sepse grave; e a taxa de mortalidade pode chegar a $55 \%$ dos pacientes que apresentam sepse nas instituições hospitalares brasileiras [9].

O estudo realizado apontou que o protocolo de SEPSE tem um alto nível de evidência, o mesmo existe para que não haja variabilidade de ações dos profissionais atuantes. Justificando-se pelo fato da sepse ser uma resposta inflamatória generalizada do organismo a um quadro infeccioso.

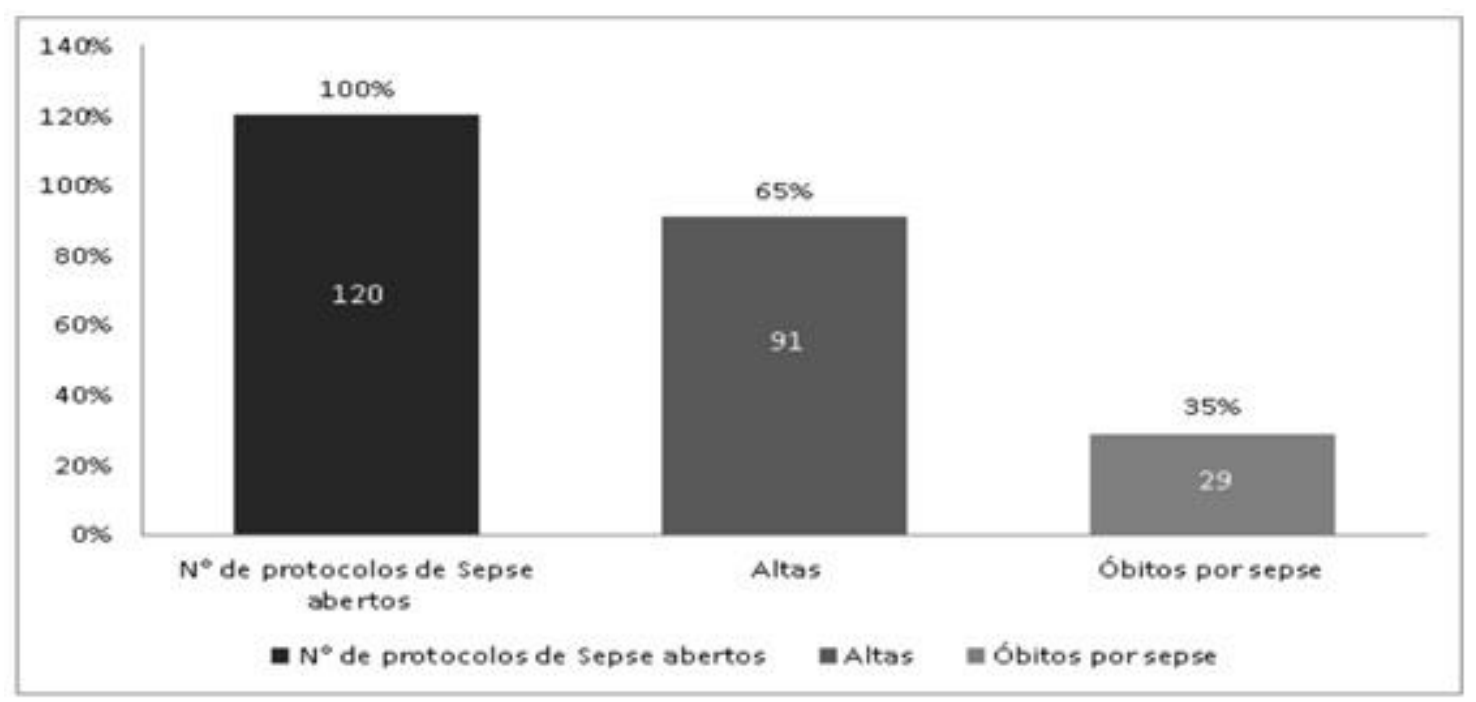

Fonte: Autor.

Figura 2 - Mortalidade por sepse em um hospital de grande porte na cidade do Rio de Janeiro, julho de 2015 a julho de 2016.

Na última década (2006 a 2016), a taxa de incidência da sepse aumentou entre $8 \%$ e $13 \%$ em relação à década passada (1995 a 2005). Muitas são as razões desse crescimento como o envelhecimento populacional, o aumento das intervenções de alto risco e o desenvolvimento de agentes infecciosos mais virulentos e resistentes a antibióticos. Em países em desenvolvimento, como o Brasil, a desnutrição, pobreza, falta de acesso a vacinas e o tratamento de forma e em tempo inadequado contribui para o aumento da mortalidade $[12,13]$. 
Ao pesquisar a mortalidade por sepse em um hospital de grande porte na cidade do Rio de Janeiro, no período de um ano, obteve-se um resultado de $35 \%$ de óbitos por sepse, mantendo sua taxa abaixo da taxa nacional que é de $65 \%$ e está dentro da média mundial que é de 30\%-40\% segundo dados do Instituto Latino Americano de Sepse. Segundo dados da comissão de revisão de óbitos do hospital onde foi realizado o estudo, foram constatados 304 óbitos e destes 29 foram por sepse. A média de mortalidade nos hospitais brasileiros é um dado significante, pois em números absolutos, o número de óbitos iguala a do infarto agudo do miocárdio e supera àqueles causados por câncer de mama e AIDS no Brasil. Os custos hospitalares atingem a cifra de 17 bilhões de reais por ano, ou 40 mil reais por paciente, por ano.

Desta forma, pode-se afirmar que sepse é uma doença de alta prevalência, elevada taxa de mortalidade e elevados custos. Nos últimos anos, evidências vêm se acumulando no sentido de mostrar a eficiência do gerenciamento do protocolo de sepse na redução de mortalidade e/ou custos institucionais [14,15].

A existência do protocolo gerenciado de sepse aumenta o resultado quanto ao sucesso terapêutico demonstrado através deste estudo no que se refere ao baixo número de óbitos por sepse comparados ao índice nacional, evidenciado pelo significativo número de altas hospitalares que foi de $65 \%$, aumentando a sobrevida e a redução dos custos assistenciais possibilitando também a credibilidade do hospital. Para alcançar esses resultados não basta apenas ter o gerenciamento do protocolo, mas garantir que todas suas etapas serão cumpridas. Portanto é fundamental a existência de uma equipe capacitada para identificar precocemente os sinais SOFA, garantindo que os exames laboratoriais sejam coletados em tempo hábil e que início da antibioticoterapia seja realizado até uma hora após a identificação dos sinais.

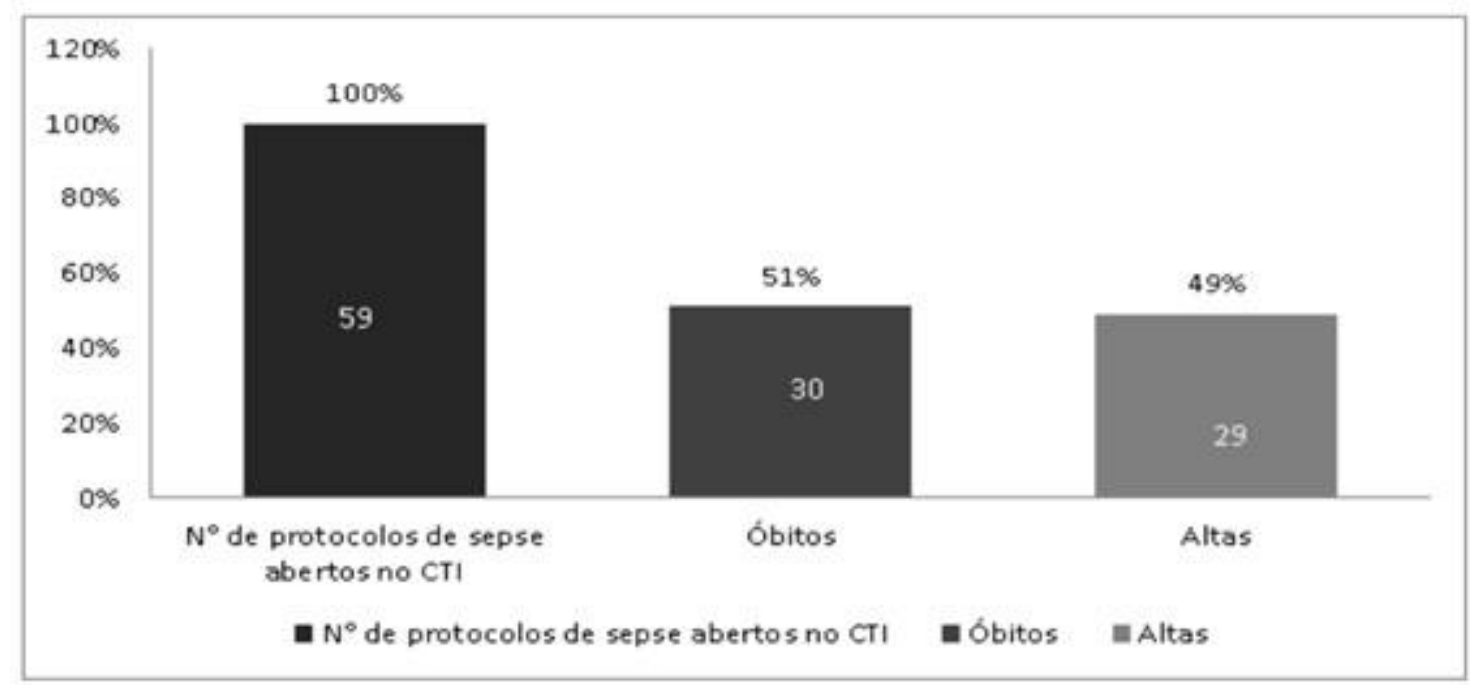

Fonte: Autor.

Figura 3 - Mortalidade por sepse no CTI de um hospital de grande porte no Rio de Janeiro após gerenciamento do protocolo de sepse, julho de 2015 a julho de 2016.

A sepse é a principal causa de morte nas unidades de terapia intensiva e está entre as principais causas de morte nos EUA. Em torno de $2 \%$ a $11 \%$ das internações hospitalares e nas Unidades de Terapia Intensiva (UTIs) são por esta doença [16]. O estudo evidenciou uma elevada mortalidade da sepse nas UTIs, dos 59 protocolos abertos de sepse, $51 \%$ evoluíram a óbito, sendo comum em nosso país segundo literaturas. Dados do Instituto Latino Americano de Sepse apontam que em torno de $50 \%$ a $60 \%$ dos pacientes internados com sepse em UTIs brasileiras morrem [9-17].

Ainda temos muito em que avançar no Brasil. A taxa de mortalidade de pacientes internados em UTI por causa da sepse é de $50 \%$ a $60 \%$ e, comparativamente com países desenvolvidos, como EUA e Canadá, a taxa varia em torno de 20\% [18].

As UTIs no Brasil precisam refletir o uso racional dos novos recursos e investir cada vez mais na educação continuada e nas discussões éticas de muitas questões na terapia intensiva, com a finalidade de reduzir os casos de óbitos por sepse, e garantir melhor sobrevida. 


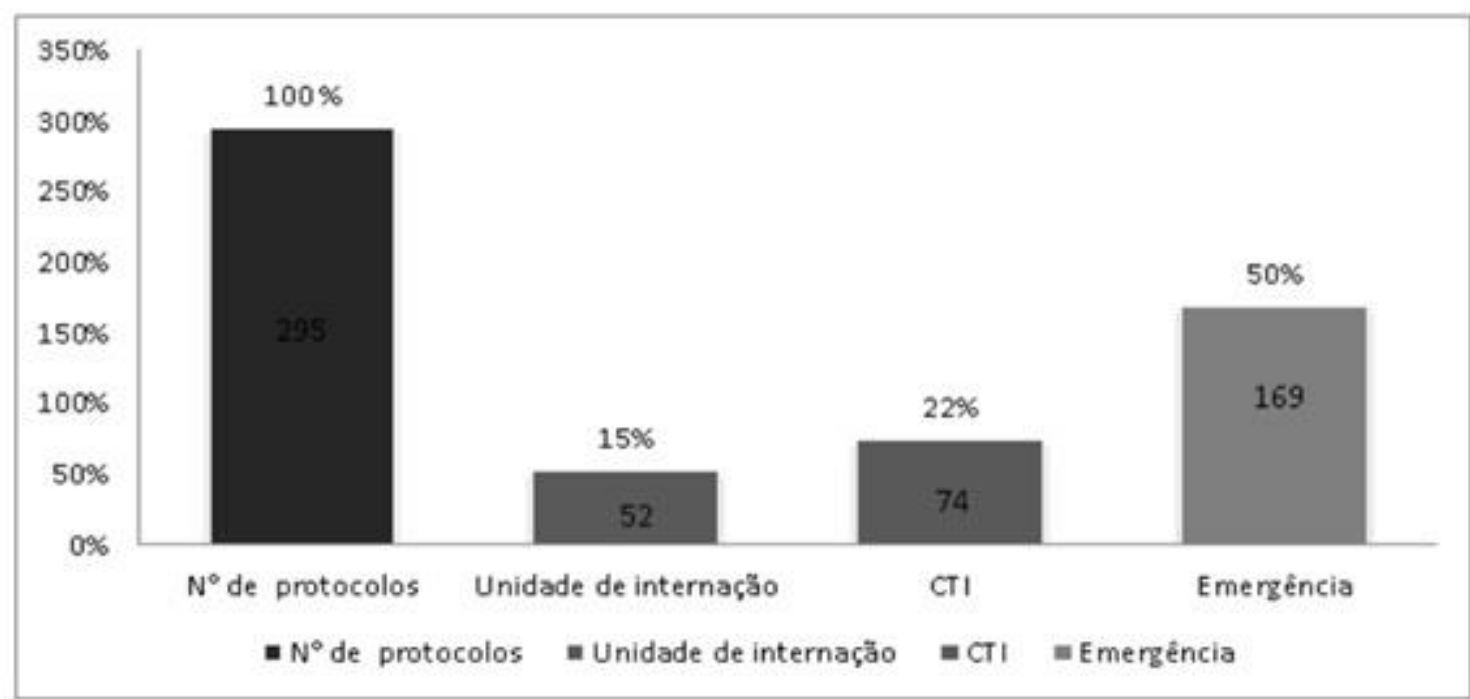

Fonte: Autor.

Figura 4 - Relação entre o número de protocolos de sepse abertos com seu local de origem, em um hospital de grande porte no Rio de Janeiro após gerenciamento do protocolo de sepse, julho de 2015 a julho de 2016.

Diversos estudos comprovam que a sepse é uma importante causa de hospitalização, e a principal causa de morte nas unidades de terapia intensiva.

Dos protocolos abertos no hospital pesquisado, 50\% foi aberto na Emergência, o que comprova que metade dos protocolos abertos nesta instituição foi de origem comunitária e que a instituição identificou precocemente os sinais, promovendo medidas assistenciais que possibilitaram melhor sobrevida proporcionada através do protocolo gerenciado. A sepse tem origem a partir de causas diferentes: a sepse comunitária tem como causa as infecções comunitárias, como pneumonias e infecções do trato urinário; a sepse hospitalar é causada por uma infecção hospitalar, geralmente proveniente de procedimentos cirúrgicos e procedimento invasivos tais como intubação, ventilação mecânica, cateterização venosa central, cateterização vesical de demora etc. Em ambas as causas, há de se instituir protocolos assistenciais de qualidade do cuidado e segurança do paciente que incluam a identificação rápida da sepse e o tratamento adequado. Além disso, é necessário a capacitação dos profissionais de saúde para implementação do protocolo em suas rotinas. A aplicação dos protocolos, que são medidas simples e de consenso internacional, aliada a campanhas de prevenção da infecção hospitalar, é o que irá mudar esse cenário de alta mortalidade por causa da sepse nos hospitais brasileiros [9-19].

\section{Conclusão}

O protocolo de sepse tem um alto nível de eficácia quando utilizado corretamente e a existência do mesmo aumenta o sucesso terapêutico e redução da mortalidade por sepse. A utilização do protocolo proporciona melhorias na qualidade assistencial garantindo uma boa assistência. O elevado número de mortes por sepse nas UTIs dado já descrito por diversas pesquisas realizadas a nível nacional e mundial afirma que a sepse é uma das principais causas de mortalidade nas UTIs. O controle rigoroso da patologia minimiza as complicações e mortalidade pela doença. Uma assistência padronizada da equipe multiprofissional diminui a variabilidade da assistência e consequentemente um tratamento com maior eficácia.

Existe uma grande quantidade de diagnosticados com sepse sendo de origem comunitária o que leva a refletir sobre a necessidade de campanhas para prevenir infecções comunitárias. A aplicação de medidas com consenso internacional, em conjunto com campanhas de prevenção da infecção hospitalar, poderá mudar o cenário de mortalidade por sepse nos hospitais brasileiros. No entanto, sugerem-se novos estudos prospectivos que abordem o impacto em longo prazo da implantação do protocolo. 
1. Koenig A. Estimativas do impacto econômico da implantação de um protocolo hospitalar para detecção e tratamento precoce de sepse grave em hospitais públicos e privados do sul do Brasil. Rev Bras Ter Intensiva 2010;22(3):213-19.

2. Pires CC. Importância do tratamento precoce na sepse grave e choque séptico: impacto no prognóstico. Rev Med Minas Gerais 2011;21(4 Supl 6):S1-S143.

3. Boechat AL. Sepse: diagnóstico e tratamento. Rev Bras Clin Med São Paulo 2010;8(5):420-7.

4. Sogayar AM, Silva ECRG, Beer IAN, Safi JJ, Kayath M. The costs group. What are the direct costs of sepsis treatment in Brazilian ICUs? Crit Care 2005;9(Suppl2):P112.

5. ANS. Implantação de diretrizes e protocolos clínicos. V1. nov. 2012. [citado 2017 Out 21]. Disponível em: http://www.ans.gov.br/images/stories/prestadores/E-EFT-01.pdf.

6. Singer M, Deutschman CS, Seymour CW, Shankar-Hari M, Annane D, Bauer M, Bellomo $\mathrm{R}$, Bernard $\mathrm{G}$ et al. The third international consensus definitions for sepsis and septic shock (Sepsis-3). JAMA 2016;315(8):801-810. http: doi:10.1001/jama. 2016. 0287.

7. Pérez AL. Clinical pathway intervention compliance and effectiveness when used in the treatment of patients with severe sepsis and septic shock at an Intensive Care Unit in Spain. Rev Latinoam Enferm 2012;20(4):635-43.

8. Barros AJP. Fundamento de metodologia cientifica. Ed. 3. São Paulo: Makron; 2007.

9. Instituto Latino Americano da Sepse. 2015. [citado 2017 out 26]. Disponível em: http://www.ilas.org.br/assets/arquivos/relatorio-nacional/relatorio-nacional.pdf

10. Rivers E, Nguyen B, Havstad S. Early Goal-Directed Therapy Collaborative Group. Early goal-directed therapy in the treatment of severe sepsis and septic shock. Engl J Med 2001;345(19):1368-77.

11. Ferrer R, Artigas A, Levy MM. Edusepsis Study Group. Improvement in process of care and outcome after a multicenter severe sepsis educational program in Spain. JAMA 2008;299(19):2294-303. http//doi: 10.1001/jama.299.19.2294.

12. Bittar DB, Pereira LV, Lemos RCA. Sistematização da assistência de enfermagem ao paciente crítico: proposta de instrumento de coleta de dados. Texto Contexto Enferm 2006;15:617-28.

13. Júnior JAL. Sepse Brasil: Estudo Epidemiológico da Sepse em Unidades de Terapia Intensiva Brasileiras. Rev Bras Ter Intensiva 2006;18(1):9-17.

14. Verena RJ, Lelivaldo ABN, Aquiles AC, Messeder OHC, Farias AMCF. Impacto clínico do diagnóstico de sepse à admissão em UTI de um hospital privado em Salvador, Bahia. J Bras Pneumol 2011;37(1):85-92.

15. Maria FCS. Revisão de literatura sobre a fisiopatologia da sepse e choque séptico. Revista UNIPLAC 2017;5(1).

16. Ricardo SPJ. Sepse: A importância do diagnóstico na emergência. Rev UNIPLAC 2016;4(1).

17. Ingrid STO. A assistência de enfermagem no diagnóstico e prevenção da sepse: revisão de literatura [Monografia]. Brasília: Faculdade de Ciências da Educação e Saúde, Centro Universitário de Brasília, Brasília, 2016. $18 f$.

18. Castro RSAP. Análise da sepse neonatal tardia em prematuros de muito baixo peso após a implantação do protocolo de sepse na unidade [Dissertação]. São Paulo: Universidade Estadual Paulista; 2017.

19. Farias LL, Pinheiro Junior FML, Braide ASG, Maieira CL, Araújo MVUM, Viana MCC. Perfil clínico e laboratorial de pacientes com sepse, sepse grave e choque séptico admitidos em uma unidade de terapia intensiva. Rev Saúde Pública 2013;6(3):50-60. 